\title{
Hyperplane arrangements between Shi and Ish
}

\author{
Rui Duarte ${ }^{1,2}$ \\ Department of Mathematics \\ University of Aveiro \\ Aveiro, Portugal \\ António Guedes de Oliveira ${ }^{1,3}$ \\ Department of Mathematics \\ Faculty of Sciences of the University of Porto \\ Porto, Portugal
}

\begin{abstract}
We introduce a new family of hyperplane arrangements in dimension $n \geq 3$ that includes both the Shi arrangement and the Ish arrangement. We prove that all the members of this family have the same number of regions - the connected components of the complement of the union of the hyperplanes - which can be bijectively labeled with the Pak-Stanley labelling. In addition, we characterise the Pak-Stanley labels of the regions of this family of hyperplane arrangements.
\end{abstract}

Keywords: Hyperplane arrangements, Shi arrangement, Ish arrangement, Pak-Stanley labelling, parking functions.

1 This work was partially supported by CMUP (UID/MAT/00144/2013) and CIDMA (UID/MAT/04106/2013), which are funded by FCT (Portugal) with national (ME) and European structural funds through the programs FEDER, under the partnership agreement PT2020.

2 Email: rduarte@ua.pt

3 Email: agoliv@fc.up.pt 


\section{Introduction}

In this paper we introduce a family of arrangements of hyperplanes in general dimension "between Ish and Shi", that is, formed by hyperplanes that are hyperplanes of the Shi arrangement or hyperplanes of the Ish arrangement, all of the same dimension, and characterise the Pak-Stanley labels of the regions of these arrangements of hyperplanes.

Consider, for an integer $n \geq 3$, hyperplanes of $\mathbb{R}^{n}$ of the following three types. Let, for $1 \leq i<j \leq n$,

$$
\begin{aligned}
& C_{i j}=\left\{\left(x_{1}, \ldots, x_{n}\right) \in \mathbb{R}^{n} \mid x_{i}=x_{j}\right\}, \\
& S_{i j}=\left\{\left(x_{1}, \ldots, x_{n}\right) \in \mathbb{R}^{n} \mid x_{i}=x_{j}+1\right\}, \\
& I_{i j}=\left\{\left(x_{1}, \ldots, x_{n}\right) \in \mathbb{R}^{n} \mid x_{1}=x_{j}+i\right\}
\end{aligned}
$$

and define, for $2 \leq k \leq n$,

$$
\begin{aligned}
\mathcal{A}_{n}^{k}:= & \left\{C_{i j} \mid 1 \leq i<j \leq n\right\} \\
& \cup\left\{I_{i j} \mid 1 \leq i<j \leq n \wedge i<k\right\} \\
& \cup\left\{S_{i j} \mid k \leq i<j \leq n\right\}
\end{aligned}
$$

Note that $\left\{C_{i j} \mid 1 \leq i<j \leq n\right\}=\operatorname{Cox}_{n}$ is the $n$-dimensional Coxeter arrangement, $\mathcal{A}_{n}^{2}=\mathrm{Shi}_{n}$ is the $n$-dimensional Shi arrangement, and $\mathcal{A}_{n}^{n}=$ $\mathrm{Ish}_{n}$ is the $n$-dimensional Ish arrangement introduced by Armstrong [2]. We represent both $\mathcal{A}_{3}^{2}=\mathrm{Shi}_{3}$ and $\mathcal{A}_{3}^{3}=\mathrm{Ish}_{3}$ on Figure 1 .

We study these arrangements with special interest in the Pak-Stanley labelings of the regions of the arrangements. The labels are $\mathcal{G}$-parking functions for special directed multi-graphs $\mathcal{G}$ as defined by Mazin [8]. In particular, we show that $\mathcal{A}_{n}^{k}$, for some $2 \leq k \leq n$, has $(n+1)^{n-1}$ regions which are bijectively labeled.

The notion of $G$-parking function was introduced by Postnikov and Shapiro in the construction of two algebras related to a general undirected graph $G$ [9]. Later, Hopkins and Perkinson [6] showed that the labels of the Pak-Stanley labeling of the regions of a given hyperplane arrangement defined by $G$ are exactly the $G$-parking functions, a fact that had been conjectured by Duval, Klivans and Martin [5]. Recently, Mazin [8] generalized this result to a very general class of hyperplane arrangements, with a similar concept based on a general directed multi-graph $\mathcal{G}$. Whereas Hopkins and Perkinson's hyperplane arrangements include for example the (original) multidimensional Shi arrangement, Mazin's hyperplane arrangements include the multidimensional 
$k$-Shi arrangement, the multidimensional Ish arrangement and in fact all the arrangements we consider here.

\section{The characteristic polynomial}

In the following, we evaluate the characteristic polynomial $\chi\left(\mathcal{A}_{n}^{k}, q\right)$.

Theorem 2.1 For every integer $2 \leq k \leq n$, the characteristic polynomial of $\mathcal{A}_{n}^{k}$ is

$$
\chi\left(\mathcal{A}_{n}^{k}, q\right)=q(q-n)^{n-1} .
$$

In terms of the arrangements $\mathcal{A}_{n}^{k}$, this means, by a celebrated result of Zaslavsky [12], that the number of regions, $r\left(\mathcal{A}_{n}^{k}\right)$, and the number of relatively bounded regions, $b\left(\mathcal{A}_{n}^{k}\right)$, do not depend on $2 \leq k \leq n$, being

$$
r\left(\mathcal{A}_{n}^{k}\right)=(-1)^{n} \chi(-1)=(n+1)^{n-1}
$$

and

$$
b\left(\mathcal{A}_{n}^{k}\right)=(-1)^{n-1} \chi(1)=(n-1)^{n-1} .
$$

These results were known (and proven similarly [1]) for both the Shi and the Ish arrangements $[3,7]$, although not for the remaining arrangements $\mathcal{A}_{n}^{k}$, with $2<k<n$.
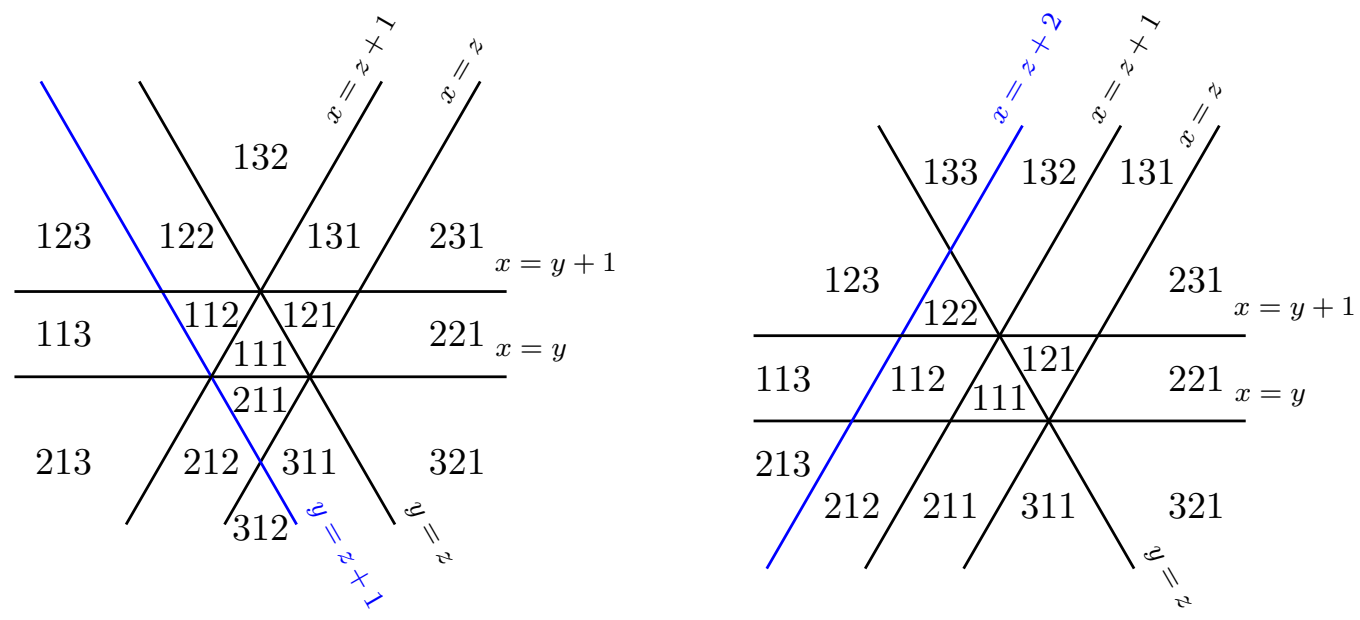

Fig. 1. Pak-Stanley labelings of $\mathcal{A}_{3}^{2}=\mathrm{Shi}_{3}$ and $\mathcal{A}_{3}^{3}=\mathrm{Ish}_{3}$ 


\section{The Pak-Stanley labelling}

Similarly to what Pak and Stanley did for the regions of the Shi arrangement (cf. [11]), we may represent a region $\mathcal{R}$ of $\mathcal{A}=\mathcal{A}_{n}^{k}$ as follows. Suppose that $\mathbf{x}=\left(x_{1}, \ldots, x_{n}\right) \in \mathcal{R}$ and $x_{w_{1}}>\cdots>x_{w_{n}}$ for a given $\mathbf{w}=\left(w_{1}, \ldots, w_{n}\right) \in \mathfrak{S}_{n}$. Let $\mathcal{H}$ be the set of triples $\left(i, j, a_{i j}\right)$ such that $i, j, a_{i j} \in \mathbb{N}, 1 \leq i<j \leq n$, $x_{i}>x_{j}$ and $a_{i j}-1<x_{i}-x_{j}<a_{i j}$. Then, of course,

$$
\mathcal{R}=\left\{\begin{array}{l|l}
\left(x_{1}, \ldots, x_{n}\right) \in \mathbb{R}^{n} & \begin{array}{l}
x_{w_{1}}>x_{w_{2}}>\cdots>x_{w_{n}}, \\
a_{i j}-1<x_{i}-x_{j}<a_{i j}, \forall\left(i, j, a_{i j}\right) \in \mathcal{H}
\end{array}
\end{array}\right\}
$$

We represent $\mathcal{R}$ by $\mathbf{w}$, decorated with one labelled arc for each hyperplane of $\mathcal{H}$, as follows. Given $\left(i, j, a_{i j}\right) \in \mathcal{H}$, the arc connects $i$ with $j$ and is labelled $a_{i j}$, with the following exceptions: if $i \leq j<p \leq m,\left(i, m, a_{i m}\right),\left(j, p, a_{j p}\right) \in \mathcal{H}$ and $a_{j p}=a_{i m}$, then we omit the arc connecting $j$ with $p$. In the Figure 2 the regions of $\mathrm{Shi}_{3}$ and $\mathrm{Ish}_{3}$ are thus represented.

The Pak-Stanley labelling of these regions may be defined as follows. As usual, let $\mathbf{e}_{i}=(0, \ldots, 0,1,0, \ldots, 0)$ be the $i$.th element of the standard basis of $\mathbb{R}^{n}$.

Definition 3.1 [Pak-Stanley labelling [11], ad.] Let $\mathcal{R}_{0}=\overbrace{2 \cdots n}$. Then label $\mathcal{R}_{0}$ with $\ell\left(\mathcal{A}_{n}^{k}, \mathcal{R}_{0}\right):=(1, \ldots, 1)$, and, given two regions $\mathcal{R}_{1}$ and $\mathcal{R}_{2}$ separated by a unique hyperplane $H$ of $\mathcal{A}_{n}^{k}$ such that $\mathcal{R}_{0}$ and $\mathcal{R}_{1}$ are on the same side of $H$, label the regions $\mathcal{R}_{1}$ and $\mathcal{R}_{2}$ so that

$\ell\left(\mathcal{A}_{n}^{k}, \mathcal{R}_{2}\right)=\ell\left(\mathcal{A}_{n}^{k}, \mathcal{R}_{1}\right)+ \begin{cases}\mathbf{e}_{i}, & \text { if } H=C_{i j} \text { for some } 1 \leq i<j \leq n \\ \mathbf{e}_{j}, & \text { if } H=S_{i j} \text { or } H=I_{i j} \text { for some } 1 \leq i<j \leq n .\end{cases}$

In the Figure 1 the Pak-Stanley labellings of the regions of $\mathrm{Shi}_{3}$ and $\mathrm{Ish}_{3}$ are shown.

\section{Partial Parking Functions}

The labels of the regions of the Shi arrangement $\mathcal{A}_{n}^{2}=\mathrm{Shi}_{n}$ form the set of $n$-dimensional parking functions, defined below, as proven by Pak and Stanley in their seminal work [10].

Definition $4.1 \mathbf{a}=\left(a_{1}, \ldots, a_{n}\right) \in[n]^{n}$ is a parking function if there is a 

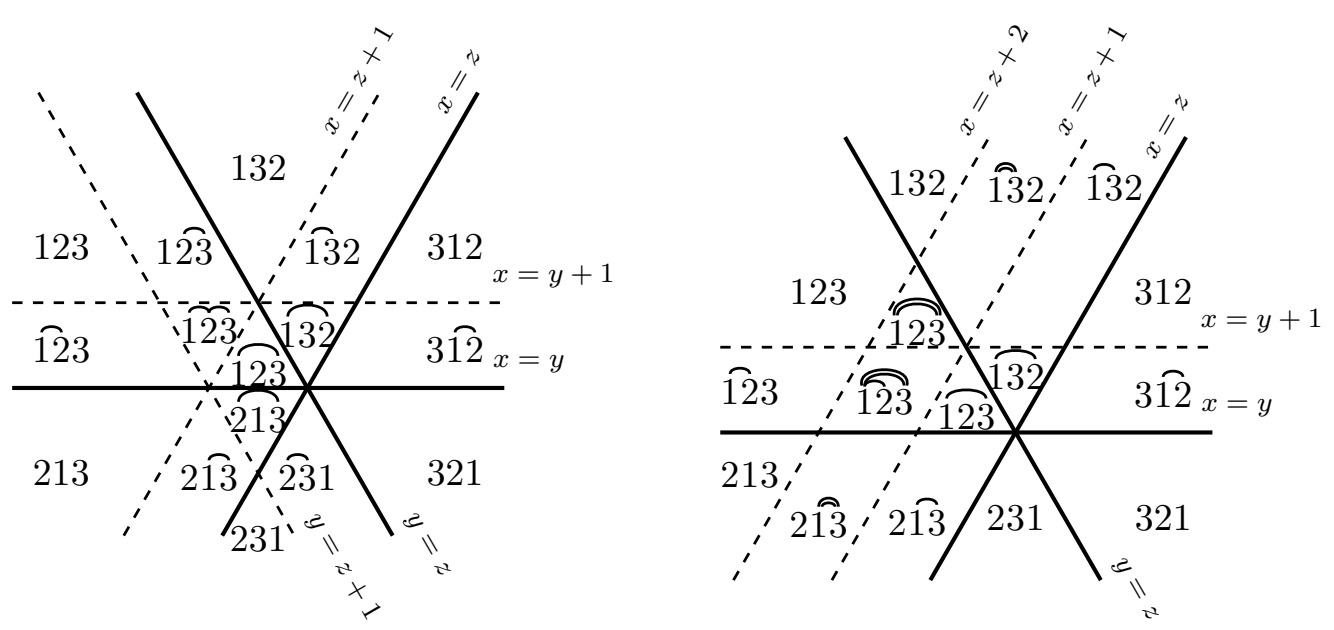

Fig. 2. $\mathrm{Shi}_{3}$ and $\mathrm{Ish}_{3}$ labelled with permutations with decorated arcs

permutation $\pi \in \mathfrak{S}_{n}$ such that

$$
a_{\pi_{i}} \leq i, \text { for every } i \in[n] .
$$

Definition 4.2 Let $\mathbf{a}=\left(a_{1}, \ldots, a_{n}\right) \in[n]^{n}$. The center of $\mathbf{a}, \mathbb{Z}(\mathbf{a})$, is the largest subset $X=\left\{x_{1}, \ldots, x_{\ell}\right\}$ of $[n]$ with $n \geq x_{1}>\cdots>x_{\ell} \geq 1$ with the property that $a_{x_{i}}<i$ for every $i \in[\ell]$.

By labelling under the same rules the regions of the $n$-dimensional Ish arrangement $\mathcal{A}_{n}^{n}=\mathrm{Ish}_{n}$, we obtain the Ish-parking functions [4] which can be characterised as follows.

Proposition $4.3 \mathbf{a}=\left(a_{1}, \ldots, a_{n}\right) \in[n]^{n}$ is an Ish-parking function if and only if $1 \in \mathbb{Z}(\mathbf{a})$.

Finally, we show that the sets of labels corresponding to the arrangements $\mathcal{A}_{n}^{k}(2 \leq k \leq n)$ that interpolate between the Shi and the Ish arrangements are the $k$-partial parking functions which are characterised as follows.

Theorem $4.4 \mathbf{a}=\left(a_{1}, \ldots, a_{n}\right) \in[n]^{n}$ is a $k$-partial parking function if and only if $1 \ldots 1 a_{k} \ldots a_{n} \in[n]^{n}$ is a parking function and there is a permutation $\pi \in \mathfrak{S}_{n}$ such that

$$
\left\{\begin{array}{l}
\pi_{i}=i, \text { for every } i \in[k-1] \\
1 \in \mathbb{Z}\left(a_{\pi_{1}} \ldots a_{\pi_{n}}\right) .
\end{array}\right.
$$




\section{References}

[1] F. Ardila, Algebraic and Geometric Methods in Enumerative Combinatorics, in Handbook of Enumerative Combinatorics, M. Bóna (ed.), Discrete Math. and Its Appl., CRC Press, Boca Raton-London-New York (2015) pp. 589-678.

[2] D. Armstrong, Hyperplane arrangements and diagonal harmonics, $J$. Combinatorics 4 (2013) pp.157-190. URL: http://dx.doi .org/10.4310/JOC. 2013.v4.n2.a2.

[3] D. Armstrong and B. Rhoades, The Shi arrangement and the Ish arrangement, Trans. Amer. Math. Soc. 364 (2012) pp. 1509-1528. URL: https://doi.org/ 10.1090/S0002-9947-2011-05521-2.

[4] R. Duarte, A. Guedes de Oliveira, The number of parking functions with center of a given length, submitted. URL: https://arxiv.org/abs/1611.03707.

[5] A. Duval, C. Klivans, and J. Martin, The G-Shi arrangement, and its relation to G-parking functions, (2011). URL: http://www.math.utep.edu/Faculty/ duval/papers/nola.pdf.

[6] S. Hopkins and D. Perkinson: Bigraphical arrangements. Trans. Amer. Math. Soc. 368 (2016) pp. 709-725. URL: http://dx.doi.org/10.1090/tran/6341.

[7] E. Leven, B. Rhoades and A. T. Wilson, Bijections for the Shi and Ish arrangements, European J. Combin. 39 (2014) pp. 1-23. URL: http://dx. doi.org/10.1016/j.ejc.2013.12.001.

[8] M. Mazin, Multigraph Hyperplane Arrangements and Parking Functions. URL: https://arxiv.org/abs/1501.01225.

[9] A. Postnikov and B. Shapiro, Trees, parking functions, syzygies, and deformations of monomial ideals, Trans. Amer. Math. Soc. 356 (2004) pp. 3109-3142. URL: https://doi.org/10.1090/S0002-9947-04-03547-0.

[10] R. P. Stanley, Hyperplane arrangements, interval orders and trees, Proc. Nat. Acad. Sci. 93 (1996) pp. 2620-2625.

[11] R. P. Stanley, An introduction to hyperplane arrangements, in: E. Miller, V. Reiner, B. Sturmfels (Eds.), Geometric Combinatorics, in: IAS/Park City Mathematics Series 13 (2007), A.M.S. pp. 389-496.

[12] T. Zaslavsky, Facing up to arrangements: face-count formulas for partitions of space by hyperplanes. Mem. Amer. Math. Soc. 1 (1975), 102 pp. URL: http: //dx.doi.org/10.1090/memo/0154. 